\title{
On Neutrosophic Semi-preopen Sets and Semi-preclosed Sets in a Neutrosophic Topological Space
}

\author{
Renu Thomas ${ }^{1 *}$, Anila. $S^{2}$ \\ ${ }^{1}$ Department of Mathematics, Sree Narayana Guru College, K.G.Chavadi, Coimbatore-105, Tamil Nadu, India \\ ${ }^{2}$ Sree Narayana Guru College, K.G.Chavadi, Coimbatore-105, Tamil Nadu, India \\ *Corresponding Author: renu7465@gmail.com Tel.:9497824758
}

Available online at: www.isroset.org

Received: 09/Sept/2018, Accepted:06/Oct/2018, Online: 31/Oct/2018

\begin{abstract}
In this paper we introduce the concept of Neutrosophic semi-preopen sets and Neutrosophic semi-preclosed sets in Neutrosophic topological spaces. After giving the fundamental definitions of neutrosophic set, operations on a neutrosophic set and neutrosophic topology, we introduce Neutrosophic semi-preopen sets and Neutrosophic semi-preclosed sets and some of their properties are derived.
\end{abstract}

Keywords: Neutrosophic set, Neutrosophic topology, Neutrosophic semi-preopen sets, Neutrosophic semi-preclosed sets.

\section{INTRODUCTION}

The concept of Fuzzy sets was introduced by Zadeh [10] in 1965 where each element had a degree of membership. Later in 1986, K. Atanassov [1,2,3] introduced the concept of Intuitionistic Fuzzy Set. It is a generalization of Fuzzy set where, besides the degree of membership, a degree of nonmembership was also assigned to each element. The Neutrosophic set was introduced by Florentin Smarandache $[4,5]$ as a generalization of Intuitionistic Fuzzy set. Later, A.A.Salama and S.A.Albowi [7,8] introduced Neutrosophic topological spaces.

This paper is organized as follows. The section 1 consists of some basic definitions and properties which are used in later sections. In section 2, we define Neutrosophic Semi-preopen sets and some of their properties are studied. The section 3 deals with the definition and properties of Neutrosophic semi- preclosed sets.

\section{PRELIMINARIES}

In this section the basic definitions for Neutrosophic sets and its operations are given.
Definition 2.1 [8] Let $X$ be a non-empty fixed set. A Neutrosophic set [NS for short] $A$ is an object having the form $\quad A=\left\{\left\langle x, \mu_{A}(x), \sigma_{A}(x), \gamma_{A}(x)\right\rangle ; x \in X\right\} \quad$ where $\mu_{A}(x), \sigma_{A}(x)$ and $\gamma_{A}(x)$ represents the degree of membership function, the degree of indeterminacy and the degree of non-membership function respectively of each element $x \in X$ to the set $A$.

Remark 2.2 [8] A Neutrosophic set $A=\left\{\left\langle x, \mu_{A}(x), \sigma_{A}(x), \gamma_{A}(x)\right\rangle\right\}$ can be identified to an

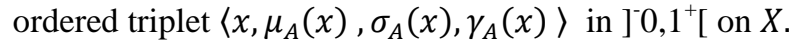

Remark 2.3 [8] For the sake of simplicity, we shall use the symbol $A=\left\langle x, \mu_{A}(x), \sigma_{A}(x), \gamma_{A}(x)\right\rangle$ for the Neutrosophic set $A=\left\{\left\langle x, \mu_{A}(x), \sigma_{A}(x), \gamma_{A}(x)\right\rangle ; x \in X\right\}$.

Remark 2.4 [8] Every intuitionistic fuzzy set (IFS) $A$ of a non empty set $X$ is a Neutrosophic set (NS) having the form $A=\left\{\left\langle x, \mu_{A}(x), 1-\left(\mu_{A}(x)+\gamma_{A}(x)\right), \gamma_{A}(x)\right\rangle ; x \in\right.$ $X\}$.

In neutrosophic set theory, the empty set $0_{\mathrm{N}}$ and full set $1_{\mathrm{N}}$ are defined as follows.

$0_{\mathrm{N}}$ may be defined as: 
$\left(0_{1}\right) 0_{\mathrm{N}}=\{\langle x, 0,0,1\rangle: x \in X\}$

$\left(0_{2}\right) 0_{\mathrm{N}}=\{\langle x, 0,1,1\rangle: x \in X\}$

$\left(0_{3}\right) 0_{\mathrm{N}}=\{\langle x, 0,1,0\rangle: x \in X\}$

$\left(0_{4}\right) 0_{\mathrm{N}}=\{\langle x, 0,0,0\rangle: x \in X\}$

$1_{\mathrm{N}}$ may be defined as:

(1 $1_{1} 1_{\mathrm{N}}=\{\langle x, 1,0,0\rangle: x \in X\}$

$\left(1_{2}\right) 1_{\mathrm{N}}=\{\langle x, 1,0,1\rangle: x \in X\}$

$\left(1_{3}\right) 1_{\mathrm{N}}=\{\langle x, 1,1,0\rangle: x \in X\}$

(14) $1_{\mathrm{N}}=\{\langle x, 1,1,1\rangle: x \in X\}$

Definition 2.5 [8] The complement of a NS $A=$ $\left\langle x, \mu_{A}(x), \sigma_{A}(x), \gamma_{A}(x)\right\rangle$ on $X$ denoted by $C(A)$ can be defined by any one of the following ways:

$\left(\mathrm{C}_{1}\right) C(A)=\left\{\left\langle x, 1-\mu_{A}(x), 1-\sigma_{A}(x), 1-\gamma_{A}(x)\right\rangle:\right.$ $x \in X\}$

$\left(\mathrm{C}_{2}\right) C(A)=\left\{\left\langle x, \gamma_{A}(x), \sigma_{A}(x), \mu_{A}(x)\right\rangle: x \in X\right\}$

$\left(\mathrm{C}_{3}\right) C(A)=\left\{\left\langle x, \gamma_{A}(x), 1-\sigma_{A}(x), \mu_{A}(x)\right\rangle: x \in X\right\}$

Definition2.6 [8] Let $A=\left\{\left\langle x, \mu_{A}(x), \sigma_{A}(x), \gamma_{A}(x)\right\rangle: x \in X\right\}$ and $B=\left\{\left\langle x, \mu_{B}(x), \sigma_{B}(x), \gamma_{B}(x)\right\rangle: x \in X\right\}$ be two neutrosophic sets of a non empty set $X$. Then we may consider two possible definitions for subsets $(A \subseteq B)$

$\mathrm{A} \subseteq \mathrm{B}$ may be defined by any one of the following ways:

(1) $A \subseteq B \Leftrightarrow \mu_{A}(x) \leq \mu_{B}(x), \sigma_{A}(x) \leq \sigma_{B}(x)$ and $\gamma_{A}(x) \geq \gamma_{B}(x) \forall x \in X$

(2) $A \subseteq B \Leftrightarrow \mu_{A}(x) \leq \mu_{B}(x), \sigma_{A}(x) \geq \sigma_{B}(x)$ and $\gamma_{A}(x) \geq \gamma_{B}(x) \forall x \in X$

Remark 2.7 [8] For any neutrosophic set A, the following conditions holds:

(1) $0_{\mathrm{N}} \subseteq A, 0_{\mathrm{N}} \subseteq 0_{\mathrm{N}}$

(2) $A \subseteq 1_{\mathrm{N}}, 1_{\mathrm{N}} \subseteq 1_{\mathrm{N}}$

Definition 2.8 [8] Let $X$ be any nonempty set, and $A=$ $\left\{\left\langle x, \mu_{A}(x), \sigma_{A}(x), \gamma_{A}(x)\right\rangle: x \in X\right\}$

and

$B=\left\langle x, \mu_{B}(x), \sigma_{B}(x), \gamma_{B}(x)\right\rangle$ be two NSs on $X$. Then,

(1) $A \cap B$ may be defined by any one of the following ways:

$$
\begin{aligned}
& (a) A \cap B= \\
& \quad\left\langle x, \mu_{A}(x) \wedge \mu_{B}(x), \sigma_{A}(x) \wedge \sigma_{B}(x) \text {, and } \gamma_{A}(x) \vee \gamma_{B}(x)\right\rangle \\
& (b) A \cap B= \\
& \quad\left\langle x, \mu_{A}(x) \wedge \mu_{B}(x), \sigma_{A}(x) \vee \sigma_{B}(x) \text {, and } \gamma_{A}(x) \vee \gamma_{B}(x)\right\rangle
\end{aligned}
$$

(2) $A \cup B$ may be defined by any one of the following ways:

(a) $A \cup B=$

$$
\left\langle x, \mu_{A}(x) \vee \mu_{B}(x), \sigma_{A}(x) \vee \sigma_{B}(x) \text {, and } \gamma_{A}(x) \wedge \gamma_{B}(x)\right\rangle
$$

(b) $A \cup B=$

$$
\left\langle x, \mu_{A}(x) \vee \mu_{B}(x), \sigma_{A}(x) \wedge \sigma_{B}(x), \text { and } \gamma_{A}(x) \wedge \gamma_{B}(x)\right\rangle
$$

The operations of intersection and union can be generalized to arbitrary family of NSs as follows:

Definition 2.9 [8]: Let $\left\{A_{i}: i \in I\right\}$ be an arbitrary family of NSs in $X$. Then,

(1) $\cap_{i \in I} A_{i}$ may be defined by any one of the following ways

(a) $\cap_{i \epsilon I} A_{i}=\left\langle x, \wedge_{i \epsilon I} \mu_{A_{i}}(x), \wedge_{i \in I} \sigma_{A_{i}}(x), \vee_{i \in I} \gamma_{A_{i}}(x)\right\rangle$

(b) $\cap_{i \epsilon I} A_{i}=\left\langle x, \wedge_{i \epsilon I} \mu_{A_{i}}(x), \vee_{i \in I} \sigma_{A_{i}}(x), \vee_{i \in I} \gamma_{A_{i}}(x)\right\rangle$

(2) $\cup_{i \in I} A_{i}$ may be defined by any of the following ways

(a) $\cup_{i \in I} A_{i}=\left\langle x, \vee_{i \in I} \mu_{A_{i}}(x), \vee_{i \in I} \sigma_{A_{i}}(x), \wedge_{i \in I} \gamma_{A_{i}}(x)\right\rangle$

(b) $\cup_{i \in I} A_{i}=\left\langle x, \vee_{i \in I} \mu_{A_{i}}(x), \wedge_{i \in I} \sigma_{A_{i}}(x), \wedge_{i \epsilon I} \gamma_{A_{i}}(x)\right\rangle$

Remark 2.10 [8] The following conditions are satisfied by any two Neutrosophic sets A and B

(1) $C(A \cap B)=C(A) \cup C(B)$

(2) $C(A \cup B)=C(A) \cap C(B)$

Definition 2.11 [8] A neutrosophic topology [NT] on a nonempty set $X$ is a family $\tau$ of neutrosophic subsets in $X$ satisfying the following axioms.

(a) $0_{N}, 1_{N} \in \tau$,

$G_{1} \cap G_{2} \in \tau$ for any $G_{1}, G_{2} \in \tau$,

$\cup G_{i} \in \tau$ for every $\left\{G_{i}: i \in J\right\} \subseteq \tau$

Then the pair $(X, \tau)$ is called a neutrosophic topological space [NTS].

Definition 2.12 [8]: Suppose $\tau$ is a neutrosophic topology on a non empty set $X$. Then elements of $\tau$ are called neutrosophic open sets [NOS] and the complement of a NOS is called a neutrosophic closed set [NCS].

Example 2.13: Let $X=\{x\}$ and $\quad A=\{\langle x, 0.3,0.7,0.8\rangle$ : $x \in X\} \quad B=\{\langle x, 0.5 .0 .6,0.3\rangle: x \in X\} \quad C=$ $\{\langle x, 0.5 .0 .7,0.3\rangle: x \in X\} \quad D=\{\langle x, 0.3 .0 .6,0.8\rangle:$ 
$x \in X\}$

be the neutrosophic

subsets of $X$.

Then, the family $\tau=\left\{0_{N}, A, B, C, D, 1_{N}\right\}$ of Neutrosophic sets in $X$ is a neutrosophic topology on $X$.

Definition 2.14 [8]: Suppose $(X, \tau)$ is a NTS and $A=$ $\left\langle x, \mu_{A}(x), \sigma_{A}(x), \gamma_{A}(x)\right\rangle$ is a NS in $X$. Then the neutrosophic closure and neutrosophic interior of $A$ is defined as follows

$\operatorname{NCl}(A)=\cap\{K: K$ is a NCS in $X$ and $A \subseteq K\}$.

$N \operatorname{Int}(A)=\cup\{G: G$ is NOS in $X$ and $G \subseteq A\}$.

$\operatorname{NCl}(A)$ is a NCS and $\operatorname{NInt}(A)$ is a NOS in $X$.

Remark 2.15 [8] For any neutrosophic set $A$ in a $\operatorname{NTS}(X, \tau)$, we have

(a) $\operatorname{NCl}(C(A))=C(\operatorname{NInt}(A))$,

(b) $\operatorname{NInt}(C(A))=C(\operatorname{NCl}(A))$.

Proposition 2.16 [8] Let $(X, \tau)$ be a NTS and $A, B$ be two NSs in $X$. Then the following conditions hold:

(a) $\operatorname{NInt}(A) \subseteq A$,

$A \subseteq N C l(A)$,

(c) $A \subseteq$

$B \Rightarrow N \operatorname{Int}(A) \subseteq N \operatorname{Int}(B)$,

(d)

$A \subseteq B \Rightarrow N C l(A) \subseteq N C l(B)$,

$N \operatorname{Int}(N \operatorname{Int}(A))=\operatorname{NInt}(A)$,

$\operatorname{NCl}(\operatorname{NCl}(A))=\operatorname{NCl}(A)$,

$N \operatorname{Int}(A \cap B)=N \operatorname{Int}(A) \cap \operatorname{NInt}(B)$,

$\operatorname{NCl}(A \cup B)=\operatorname{NCl}(A) \cup \operatorname{NCl}(B)$,

$\operatorname{NInt}\left(0_{N}\right)=0_{N}$,

$\operatorname{NInt}\left(1_{N}\right)=1_{N}$,

(k) $N C l\left(0_{N}\right)=0_{N}$,

(l) $\operatorname{NCl}\left(1_{N}\right)=1_{N}$,

$A \subseteq B \Rightarrow \mathrm{C}(B) \subseteq C(A)$,

$B) \subseteq N C l(A) \cap N C l(B)$,

$B) \supseteq N \operatorname{Int}(A) \cup N \operatorname{Int}(B)$.

Definition 2.17: [6,9] Let $X$ be a NTS and $A$ be a NS of $X$. Then,

(a) $A$ is said to be a neutrosophic semi open [NSO] set if and only if $A \subseteq \operatorname{NCl}(\operatorname{Nint}(A))$

(b) $A$ is said to be neutrosophic semi closed [NSC] set if and only if $\operatorname{NInt}(\operatorname{NCl}(A)) \subseteq A$ (c) $A$ is said to be a neutrosophic preopen [NPO] set if and only if $A \subseteq \operatorname{NInt}(\operatorname{NCl}(A))$

(d) $A$ is said to be a neutrosophic preclosed [NPC] set if and only if $\operatorname{NCl}(\operatorname{NInt}(A)) \subseteq A$

\section{NEUTROSOPHIC SEMI-PREOPEN SET IN A NEUTROSOPHIC TOPOLOGICAL SPACE.}

In this section, the neutrosophic semi-preopen set is introduced and their properties are studied.

Definition 3.1: Let $X$ be a NTS and $A$ be a NS in $X$. Then, $A$ is said to be a neutrosophic semi-preopen [NSPO] set of $X$ if and only if $A \subseteq N C l(N \operatorname{Int}(N C l(A)))$.

Theorem 3.2 Let $(X, \tau)$ be a NTS. Then the union of any two NSPO sets in $X$ is also a NSPO set in $X$.

Proof: Let $A$ and $B$ be any two NSPO sets of $X$.

Then, $A \subseteq \operatorname{NCl}(\operatorname{NInt}(\operatorname{NCl}(A)))$ and

$B \subseteq \operatorname{NCl}(\operatorname{NInt}(\operatorname{NCl}(B))) \quad \Rightarrow A \cup$

$B \subseteq N C l(N \operatorname{Int}(N C l(A))) \cup \operatorname{NCl}(\operatorname{NInt}(N C l(B)))$

$=\operatorname{NCl}(\operatorname{NInt}(N \operatorname{Cl}(A)) \cup \operatorname{NInt}(N C l(B)))$

[by proposition $1.16(h)$ ]

$\subseteq \operatorname{NCl}(\operatorname{NInt}(\operatorname{NCl}(A) \cup \operatorname{NCl}(B)))$

[by proposition $1.16(\mathrm{o})$ ]

$=\operatorname{NCl}(\operatorname{NInt}(\operatorname{NCl}(A \cup B)))$

[by proposition $1.16(h)$ ]

$\therefore A \cup B \subseteq N C l(N \operatorname{Nint}(N C l(A \cup B)))$.

Therefore $A \cup B$ is a NSPO set in $X$.

Corollary 3.3 Let $(X, \tau)$ be a NTS. If $\left\{A_{\alpha}\right\}_{\alpha \in \Delta}$ is a collection of NSPO sets in $X$. Then, $\mathrm{U}_{\alpha \epsilon \Delta} A_{\alpha}$ is a NSPO set in $X$.

Proof: Let $A_{\alpha}$ be a NSPO set for each $\alpha \in \Delta$. Then for each $\quad \alpha \in \Delta, \quad A_{\alpha} \subseteq \operatorname{NCl}\left(\operatorname{NInt}\left(\operatorname{NCl}\left(A_{\alpha}\right)\right)\right) \Rightarrow$

$\mathrm{U}_{\alpha \epsilon \Delta} A_{\alpha} \subseteq \mathrm{U}_{\alpha \in \Delta} \operatorname{NCl}\left(\operatorname{NInt}\left(\operatorname{NCl}\left(A_{\alpha}\right)\right)\right) \quad=$ $\operatorname{NCl}\left(\mathrm{U}_{\alpha \in \Delta} \operatorname{NInt}\left(\operatorname{NCl}\left(A_{\alpha}\right)\right)\right)$

[by proposition $1.16(h)$ ]

$\subseteq \operatorname{NCl}\left(\operatorname{NInt}\left(\mathrm{U}_{\alpha \epsilon \Delta \Delta} \operatorname{NCl}\left(A_{\alpha}\right)\right)\right)$

[by proposition $1.16(o)$ ]

$=\operatorname{NCl}\left(\operatorname{NInt}\left(\operatorname{Ncl}\left(\cup_{\alpha \in \Delta} A_{\alpha}\right)\right)\right)$ 
[by proposition $1.16(h)]$

$\therefore \cup_{\alpha \in \Delta} A_{\alpha} \subseteq \operatorname{NCl}\left(\operatorname{NInt}\left(\operatorname{Ncl}\left(\mathrm{U}_{\alpha \in \Delta} A_{\alpha}\right)\right)\right)$.

Therefore, $\mathrm{U}_{\alpha \in \Delta} A_{\alpha}$ is a NSPO set in $X$.

Remark 3.4: The intersection of any two NSPO sets of a neutrosophic topological space need not be a NSPO set as shown in the following example.

Example 3.5: Let $X=\{a, b\}$ and

$A=\{\langle 0.3,0.2,0.7\rangle\langle 0.4,0.1,0.5\rangle\}$

$B=\{\langle 0.2,0.3,0.4\rangle\langle 0.5,0.4,0.5\rangle\}$

$C=\{\langle 0.3,0.3,0.4\rangle\langle 0.5,0.4,0.5\rangle\}$

$D=\{\langle 0.2,0.2,0.7\rangle\langle 0.4,0.1,0.5\rangle\}$

Then $\tau=\left\{0_{N}, A, B, C, D, 1_{N}\right\}$ is a NTS on $X$. Let

$A_{1}=\{\langle 0.8,0.1,0.5\rangle\langle 0.4,0.2,0.7\rangle\}$ and

$A_{2}=\{\langle 0.5,0.2,0.3\rangle\langle 0.6,0.5,0.3\rangle\}$.

Here, $N C l\left(N \operatorname{Int}\left(\operatorname{NInt}\left(A_{1}\right)\right)\right)=1_{N}$

and $\operatorname{NCl}\left(\operatorname{NInt}\left(\operatorname{NCl}\left(A_{2}\right)\right)\right)=1_{N}$.

Therefore, $A_{1}$ and $A_{2}$ are NSPO sets of $X$.

But $A_{1} \cap A_{2}=\{\langle 0.5,0.1,0.5\rangle\langle 0.4,0.2,0.7\rangle\}$ is not a NSPO set in $X$.

Theorem 3.6: Let $A$ be a NSPO set in a NTS $X$ and suppose $A \subseteq B \subseteq N C l(A)$. Then $B$ is a NSPO set in $X$.

Proof: $A$ is a NSPO set.

$$
\begin{aligned}
\therefore \quad A \subseteq \operatorname{NCl} & (\operatorname{NInt}(\operatorname{NCl}(A))) \\
\Rightarrow \operatorname{NCl}(A) & \subseteq \operatorname{NCl}(\operatorname{NCl}(\operatorname{NInt}(\operatorname{NCl}(A)))) \\
& =\operatorname{NCl}(\operatorname{NInt}(\operatorname{NCl}(A)))
\end{aligned}
$$

[by proposition $1.16(f)$ ]

$\therefore \operatorname{NCl}(A) \subseteq \operatorname{NCl}(\operatorname{NInt}(\operatorname{NCl}(A)))$

Given, $A \subseteq B \subseteq N C l(A)$. Hence it follows that

$B \subseteq \operatorname{NCl}(\operatorname{NInt}(\operatorname{NCl}(A)))$.

We have $A \subseteq B$

$\therefore \operatorname{NCl}(\operatorname{NInt}(\operatorname{NCl}(A))) \subseteq \operatorname{NCl}(\operatorname{NInt}(\operatorname{NCl}(B)))$

[ by proposition $1.16(c)$ and $1.16(d)$ ]

$\therefore B \subseteq N C l(N \operatorname{Nnt}(\operatorname{NCl}(B)))$.

Hence

$B$ is a NSPO set.

Theorem 3.7: Every NPO set in a NTS $X$ is a NSPO set.

Proof: Let $A$ be a NPO set.

Then

$A \subseteq N \operatorname{Int}(\operatorname{NCl}(A))$
$\operatorname{NCl}(A) \subseteq N C l(\operatorname{NInt}(\operatorname{NCl}(A)))$

have $A \subseteq \operatorname{NCl}(A)$

[by proposition $1.16(d)] \mathrm{We}$

$A \subseteq N C l(N \operatorname{Nnt}(N C l(A)))$

Therefore,

NSPO set in $X$.

Remark 3.8: Converse of the above theorem need not be true as shown in the example below.

Example 3.9: Let $X=\{a, b\}$ and

$A=\{\langle 0.3,0.2,0.7\rangle\langle 0.4,0.1,0.5\rangle\}$

$B=\{\langle 0.2,0.3,0.4\rangle\langle 0.5,0.4,0.5\rangle\}$

$C=\{\langle 0.3,0.3,0.4\rangle\langle 0.5,0.4,0.5\rangle\}$

$D=\{\langle 0.2,0.2,0.7\rangle\langle 0.4,0.1,0.5\rangle\}$

Then $\tau=\left\{0_{N}, A, B, C, D, 1_{N}\right\}$ is a NTS on $X$. Let $P=\{\langle 0.3,0.5,0.4\rangle\langle 0.3,0.4,0.6\rangle\}$

Then, $P$ is a NSPO set in $X$ but not a NPO set.

Theorem 3.10: Every NSO set in a NTS $X$ is a NSPO set in $X$.

Proof: Suppose $A$ is a NSO set.

Then, $A \subseteq N C l(N \operatorname{Int}(A))$

we have $A \subseteq \operatorname{NCl}(A)$

$\therefore \operatorname{NCl}(\operatorname{NInt}(A)) \subseteq \operatorname{NCl}(\operatorname{NInt}(\operatorname{NCl}(A)))$

[by proposition $1.16(c)$ and $1.16(d)$ ]

Hence it follows that $A \subseteq \operatorname{NCl}(\operatorname{NInt}(\operatorname{NCl}(A)))$

$\therefore A$ is a NSPO set.

Remark 3.11: Converse of the above theorem need not be true as shown in the following example:

Example 3.12: Let $X=\{a, b\}$ with

$A=\{\langle 0.3,0.5,0.4\rangle\langle 0.6,0.2,0.5\rangle\}$

$B=\{\langle 0.2,0.6,0.7\rangle\langle 0.5,0.3,0.1\rangle\}$

$C=\{\langle 0.3,0.6,0.4\rangle\langle 0.6,0.3,0.1\rangle\}$

$D=\{\langle 0.2,0.5,0.7\rangle\langle 0.5,0.2,0.5\rangle\}$ $\tau=$

$\left\{0_{N}, A, B, C, D, 1_{N}\right\}$ is a neutrosophic topology on $X$.

Then, $P=\{\langle 0.4,0.6,0.4\rangle\langle 0.5,0.3,0.4\rangle\}$ is a NSPO set but not a NSO set.

Theorem 3.13: Every NOS in a NTS $X$ is a NSPO set in $X$.

Proof: Suppose $A$ is a NOS in X. As every NOS is a NSO set, from theorem 3.10 it easily follows that $A$ is a NSPO set. 
Remark 3.14: Converse of the above theorem need not be true as shown in the example below:

Example 3.15: Let $X=\{a, b, c\}$ with

$A=\{\langle 0.4,0.5,0.2\rangle\langle 0.3,0.2,0.1\rangle\langle 0.9,0.6,0.8\rangle\}$

$B=\{\langle 0.2,0.4,0.5\rangle\langle 0.1,0.1,0.2\rangle\langle 0.6,0.5,0.8\rangle\}$ $\tau=$

$\left\{0_{N}, A, B, 1_{N}\right\}$ is a neutrosophic topology on $X$. Then, $C=\{\langle 0.5,0.6,0.1\rangle\langle 0.4,0.3,0.1\rangle\langle 0.9,0.8,0.5\rangle\}$ is a NSPO set but not a NSO set.

\section{NEUTROSOPHIC SEMI-PRECLOSED SETS IN NEUTROSOPHIC TOPOLOGICAL SPACES.}

In this section we introduce the concepts of the neutrosophic semi-preclosed sets and some of their properties are studied.

Definition 4.1: A neutrosophic set $A$ of a NTS $X$ is aid to be a neutrosophic semi-preclosed set in $X$ if and only if $N \operatorname{Int}(N C l(N \operatorname{Int}(A))) \subseteq A$.

Theorem 4.2: Let $(X, \tau)$ be a NTS and $A$ be a NS of $X$. Then, $A$ is a NSPC set if and only if $C(A)$ is a NSPO set.

Proof: Suppose $A$ is a NSPC set in $X$. Then,

$\operatorname{NInt}(\operatorname{NCl}(\operatorname{NInt}(A))) \subseteq A$

taking

compliments on both sides, we get

$C(\operatorname{NInt}(\operatorname{NCl}(\operatorname{NInt}(A))))$ $C(A) \subseteq$

$\operatorname{NCl}(\operatorname{NInt}(\operatorname{NCl}(C(A))))$

[by remark 1.15]

$\therefore C(A) \subseteq N C l(N \operatorname{Nint}(\operatorname{NCl}(C(A))))$

hence $C(A)$ is NSPO set in $X$.

Conversely suppose $C(A)$ is a NSPO set in $X$. Then,

$C(A) \subseteq N C l(N \operatorname{Int}(N C l(C(A))))$

taking compliments on both sides, we get

$C(\operatorname{NCl}(\operatorname{NInt}(\operatorname{NCl}(C(A))))) \subseteq A$

$\Rightarrow \quad N \operatorname{Int}(N \operatorname{Nl}(\operatorname{NInt}(A))) \subseteq A$

[by remark 1.15]

hence it follows that $A$ is a NSPC set in $X$.

Theorem 4.3: Let $(X, \tau)$ be a NTS. Then, the intersection of any two NSPC sets in $X$ is also a NSPC set.
Proof: Let $A$ and $B$ be two NSPC sets in $X$. Then,

$N \operatorname{Int}(N C l(N \operatorname{Int}(A))) \subseteq A$ and

$N \operatorname{Int}(N C l(N \operatorname{Int}(B))) \subseteq B$

Therefore,

$N \operatorname{Int}(N C l(N \operatorname{Int}(A))) \cap N \operatorname{Int}(N \operatorname{Cl}(N \operatorname{Int}(B))) \subseteq A \cap B$

now by using proposition $1.16(\mathrm{~g})$ and $1.16(\mathrm{n})$, we get

$N \operatorname{Int}(N C l(N \operatorname{Int}(A \cap B))) \subseteq A \cap B$.

Hence, $A \cap B$ is a NSPC set.

Remark 4.4: The union of two NSPC sets in a NTS $X$ need not be a NSPC as shown in the following example.

Example 4.5: Let $X=\{a\}$ with

$A=\{\langle 0.2,0.5,0.3\rangle\}$

$B=$

$\{\langle 0.1,0.5,0.7\rangle\}$.

Then, $\tau=\left\{0_{N}, A, B, 1_{N}\right\}$ is a neutrosophic topology on $X$. Let $A_{1}=\{\langle 0,0.5,0.8\rangle\}$ and $A_{2}=\{\langle 0.1,0.2,0.3\rangle\}$.

$\operatorname{NInt}\left(A_{1}\right)=0_{N}$ and $\operatorname{NInt}\left(A_{2}\right)=0_{N}$.

Hence, $A_{1}$ and $A_{2}$ are NSPC sets in $X$ but $A_{1} \cup A_{2}$ is not a NSPC set.

Theorem 4.6: Every NPC set in a NTS $X$ is a NSPC set.

Proof: Suppose $A$ is a NPC set.

Then, $\operatorname{NCl}(\operatorname{NInt}(A)) \subseteq A$.

Now, by using proposition 1.16 (c), we get

$N \operatorname{Int}(N C l(N \operatorname{Int}(A))) \subseteq N \operatorname{Int}(A)$

we have $\operatorname{NInt}(A) \subseteq A$.

Hence it follows that $N \operatorname{Int}(N \operatorname{Cl}(\operatorname{NInt}(A))) \subseteq A$

Thus we get that $A$ is a NSPC set.

Remark 4.7: The converse of the above theorem need not be true as shown by the following example.

Example 4.8: Let $X=\{a\}$ with

$A=\{\langle 0.4,0.5,0.3\rangle\}, B=\{\langle 0.1,0.5,0.5\rangle\}$.

Then, $\tau=\left\{0_{N}, A, B, 1_{N}\right\}$ is a neutrosophic topology on $X$. Let $C=\{\langle 0.3,0.6,0.5\rangle\}$. Then, $C$ is a NSPC set but not a NPC set.

Theorem 4.9: Every NSC set in a NTS $X$ is a NSPC set.

Proof: Suppose $A$ is a NSC set in $X$.

Then, $\operatorname{NInt}(\operatorname{NCl}(A)) \subseteq A$.

We have $\operatorname{NInt}(A) \subseteq A$.

Therefore by using proposition $1.16(\mathrm{c})$ and $1.16(\mathrm{~d})$, it 
follows that

$$
\begin{aligned}
& N \operatorname{Int}(\operatorname{NCl}(\operatorname{NInt}(A))) \subseteq \operatorname{NInt}(\operatorname{NCl}(A)) \quad \text { Therefore, } \\
& N \operatorname{Int}(\operatorname{NCl}(\operatorname{NInt}(A))) \subseteq A . \\
& \text { that } A \text { is a NSPC set. }
\end{aligned}
$$

Remark 4.10: The converse of the above theorem need not be true as shown in the example below.

Example 4.11: Let $X=\{a\}$ with

$A=\{\langle 0.2,0.5,0.3\rangle\}, B=\{\langle 0.1,0.5,0.7\rangle\}$.

Then, $\tau=\left\{0_{N}, A, B, 1_{N}\right\}$ is a neutrosophic topology on $X$. Let $A_{1}=\{\langle 0,0.5,0.8\rangle\} . A_{1}$ is a NSPC set but not a NSC set.

Theorem 4.12: Every NCS in $X$ is a NSPC set.

Proof: Suppose $A$ is a NCS set in $A$. As every NCS set is a NSC set, it follows from the above theorem 4.9 that $A$ is a NSPC set

Remark 4.13: The converse of the above theorem need not be true as shown in the following example.

Example 4.14: Let $X=\{a, b, c\}$ with

$A=\{\langle 0.5,0.6,0.3\rangle\langle 0.1,0.7,0.9\rangle\langle 1,0.6,0.4\rangle\}$

$B=\{\langle 0,0.4,0.7\rangle\langle 0.1,0.6,0.9\rangle\langle 0.5,0.5,0.8\rangle\} \quad \tau=$

$\left\{0_{N}, A, B, 1_{N}\right\}$ is a neutrosophic topology on $X$. Then, $C=\{\langle 0.2,0.4,0.9\rangle\langle 0,0.2,0.9\rangle\langle 0.3,0.2,1\rangle\}$ is a NSPC set but not a NCS.

Theorem 4.15: If $A$ is NSPC set in a NTS $X$ and suppose $\operatorname{NInt}(A) \subseteq B \subseteq A$, then $B$ is also a NSPC set in $X$.

Proof: $A$ is a NSPC set. $\therefore N \operatorname{Int}(N C l(N \operatorname{Int}(A))) \subseteq A$

Then by proposition 1.16 (c) and 1.16 (e) we get

$N \operatorname{Int}(\operatorname{NCl}(\operatorname{NInt}(A))) \subseteq N \operatorname{Nint}(A)$.

Given $N \operatorname{Int}(A) \subseteq B$.

Hence it follows that $N \operatorname{Int}(\operatorname{NCl}(\operatorname{NInt}(A))) \subseteq B$.

Also, $B \subseteq A$. $\therefore$ by using proposition 1.16 (c) and 1.16 (d)

$N \operatorname{Int}(\operatorname{NCl}(\operatorname{NInt}(B))) \subseteq N \operatorname{Int}(\operatorname{NCl}(\operatorname{NInt}(A)))$

Thus, NInt $(N C l(N \operatorname{Int}(B))) \subseteq B$

Hence $B$ is a NSPC set.

\section{CONCLUSION}

The basic aim of this paper is the introduction of two new sets - the semi-preopen set and the semi-preclosed set in a neutrosophic topological space. Then certain properties of these sets have been studied in detail.

\section{REFERENCES}

[1]. K.Atanassov, Intuitionistic fuzzy sets, Fuzzy Sets and Systems 1986: 20: 87-96.

[2]. K.Atanassov, Intuitionistic fuzzy sets, in V.Sgurev, ed., vii ITKRS Session, Sofia (June 1983 central Sci. and Techn. Library, Bulg.Academy of Sciences (1984)).

[3]. K.Atanassov, Review and new result on intuitionistic fuzzy sets, preprint IM-MFAIS-1-88, 1988.

[4]. Florentine Smarandache A Unifying Field in Logic, Neutrosophic Logic, Neutrosophy, Neutrosophic Set, Neutrosophic Probability, American Research Press, Rehoboth, NM, 1999.

[5]. Florentine Smarandache, Neutrosophy and Neutrosophic Logic, First International Conference on Neutrosophy, Neutrosophic Logic, Set, Probability and statistics, University of New Mexico, Gallup, NM 87301, USA(2002), smarand@unm.edu.

[6]. P. Iswarya., Dr. K. Bageerathi, On Neutrosophic Semi-open Sets in Neutrosophic Topological Spaces, IJMTT, Vol (37) Number 3 -Sep 2016 214-223.

[7]. A.A.Salama and S.A.Albowi, Generalized Neutrosophic sets and Generalized Neutrosophic topological spaces, Journal of Computer science and Engineering Vol(2) no:7 (2012)

[8]. A.A.Salama and S.A.Albowi, Neutrosophic set and Neutrosophic Topological spaces, IOSR.JM. Vol (3) (2012), 31-35.

[9]. V.Venkateswara Rao, Y. Srinivasa Rao, Neutrosophic Preopen sets and Preclosed sets in Neutrosophic Topological spaces, International journal of ChemTech Research, Vol 10 No: $10,449-458$

[10]. L.A.Zadeh, Fuzzy sets, Inform and Control8 (1965), 338353.

\section{AUTHORS PROFILE}

Ms. Renu Thomas, is currently working as assistant professor and Head at Department of Mathematics, Sree Narayana Guru College, K.G.Chavadi, Coimbatoer-105, Tamil Nadu. She has submitted dissertation and is awaiting Ph.D. degree. The author has also published 18 papers in various international journals. 